\title{
An Application of the Visualization Methods for Investigation Small Scale Processes in the Atmosphere-hydrosphere Boundary Layer within Laboratory Experiments on the Wind-wave Facilities
}

\author{
Daniil Sergeev ${ }^{1}$, Anatoly Suvorov ${ }^{1}$ and Alexander Kandaurov ${ }^{1}$ \\ ${ }^{1}$ Federal Research Center Institute of Applied Physics of the Russian Academy of Sciences, 46 Ul'yanov Street, \\ Nizhny Novgorod, 603950, Russia
}

\begin{abstract}
Hydro/aerodynamic laboratory experiment aimed at the laboratory modelling of the physical processes marine atmospheric boundary layer is one of the most complicated. Especial features as spray of droplets, the bubbles in the water and foam generated during the breaking of the waves should be taken into account when modelling extreme weather conditions caused by severe winds. Thus, in the experiment we are dealing with a multiphase turbulent flow with a free boundary. This investigation describes developing approaches to the use of optical methods based on visualization for performing these investigations. Presented results were obtained in experiments carried out on wind-wave facilities. To study the processes of fragmentation of the water surface leading to the formation of droplets and foam, high-speed multi-angle video taking is used in combination with the shadow imaging method.
\end{abstract}

\section{Keywords}

wind, waves, spray, foam, laboratory modeling, visualization

\section{Introduction}

The boundary layer between the atmosphere and hydrosphere (first of all the world ocean is meaning) of our planet is characterized by a large number of different processes physical processes (see review [1]). It is clear from it that all physical processes in the atmosphere and hydrophysical processes of the ocean cannot be separated from each other, and they are closely interconnected. Regular wave breaking leads to formation of whitecap zones and foam crests, the generation of sprays and the involvement of air in water with the formation of bubbles that float and break the surface, also lead to splashing. Spray significantly intensifies heat transfer compared to a smooth water surface. Foam, also formed during the breaking of waves, also strongly effects on the heat and momentum transfer in the boundary layer. It is obvious that from the point of view of the tasks of experimental research of the processes of interaction between the hydrosphere and atmosphere, the main problem is the need to simultaneously measure as many physical characteristics as possible with high spatial and temporal resolution, in a wide range of conditions including extreme (storms, hurricanes, etc.). it is quite difficult to solve this problem during in situ measurements. Therefore it is actively resorted to laboratory modeling, creating large-scale experimental facilities - wind-wave tanks and flumes. In addition to realizing the conditions, it is also necessary to provide measurements of various quantities characterizing physical processes in the air flow, at the interface and processes in the water layer. From the point of view of a laboratory aero/hydrodynamic experiment, it can be concluded that it is necessary to study the turbulent process of wind-wave interaction, dealing with a complex dynamic interface between the liquid (water) and gas (air) phases. Among the main problems, it should be highlighted: 1)

GraphiCon 2021: 31st International Conference on Computer Graphics and Vision, September 27-30, 2021, Nizhny Novgorod, Russia EMAIL: daniil@ipfran.ru (D. Sergeev); suvorov@appl.sci-nnov.ru (A. Suvorov) green.pb@gmail.com (A. Kandaurov); ORCID: 0000-0003-4910-3935 (D. Sergeev); 0000-0002-0014-8887 (A. Kandaurov);

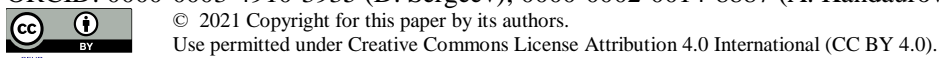


the need for measurements in air and water close to the interface, which varies significantly in space and time depending on the characteristics of the wind and waves 2) the need for measurements with high spatial and temporal resolution to obtain the necessary ensemble of data for subsequent statistical processing 3) the multiphase nature of the air and water flows due to the formation of sprays and underwater bubbles in strong winds with regular breaking waves. Optical methods based on visualization of flows compares favorably point measurements. It allows simultaneous observations in significant volumes of continuous media with high spatial resolution, and also do not introduce disturbances into the medium under study. The present work is devoted to the processes of interaction of the air flow with a rough water surface under laboratory modeling of extreme weather conditions, primarily associated with strong winds. Field measurements in such conditions are very difficult. That is why this study with laboratory modeling focused on the measurements of sprays and foam coverage which are the features of the boundary layer under severe conditions, the measurements of which are often more complicated than the measurements of the characteristics of flows in the surface water layer. At high winds regular wave breaking begins with the fragmentation of the water surface, leading to the generation of sprays and the formation of foam on the surface. The first part of the work is devoted to the study of the processes of generation of spray droplets from the rough surface, and their characteristics. The second is to study the characteristics of the foam and methods for its artificial creation in laboratory experiments (the results of recent studies are presented).

\section{Investigation of spray within laboratory modelling of the interaction between atmosphere and hydrosphere ding}

Sea spray is a very important environmental effect and a common feature of the marine atmospheric boundary layer. The size distribution of droplets injected in a unit of time from a unit of water surface is quantitively characterized by sea spray generation function (SSGF). Problems of direct measurements of droplets parameters in severe conditions and lack of understanding about the ways of the spray formation are the main causes for insufficient knowledge on SSGF leading to significant uncertainties in estimations of sea sprays influence on the marine atmospheric boundary layer [2,3]. Even the spraygeneration mechanisms in extreme winds remained undetermined up to present time. Such difficulties are primarily associated with the problems of obtaining a sufficient amount of empirical data not only in field, but also in laboratory modeling. Different methods used in experiments can be divided on point (see for example [4-6]) and volumetric methods [7-9]. Volumetric methods are based on visualization: illumination with a laser sheet [7] or shadowgraph method $[8,9]$ with video recording, including highspeed filming, which became very popular. It allows to obtain the data of the spray characteristics and to study in detail the process of its formation both. It should be noted that regardless of the methods used, measuring the characteristics of spray (velocity, size) near the water surface especially for high and strongly breaking waves caused by severe wind is a very difficult task.

The main feature of the studies performed by our group in the last three years ([9]) was combination shadow visualization method with multi- view high speed and resolution filming of the water surface (see Figure $1 \mathrm{a}$ ). Investigations were carried out on a unique experimental setup - Thermostratified Wind-Wave Tank (TSWiWaT) of IAP RAS. This level is a highest for the present time in experiments on the study of sprays in the conditions of laboratory modeling wind-wave interaction. Basing on the results of [9], the classification of phenomena (projections, bursting bubbles, bagbreakup) leading to the generation of sprays was clarified. It was possible to obtain statistics of precisely the phenomena leading to the generation of splashes due to the additional filming of a top view of the water surface illuminated by underwater lights, which was carried out for the first time. It demonstrated that the bag breakup mechanism is dominant at severe wind conditions (see Figure $1 \mathrm{~b}$ ).

The obtained statistics data allowed us to offer a fundamentally new approach to obtaining the necessary SSGF. It can be obtained as a combination (convolution) of the statistics of phenomena leading to the sprays formation and statistics of droplets occurred in a single phenomenon (see [10]). At the same time, it is rather difficult to study in detail a single phenomenon under natural conditions of wind-wave interaction, even in laboratory modeling, given its sporadic nature and the difficulty of performing measurements, especially at high wind speeds. Therefore, the idea was proposed to artificially initiate these phenomena under controlled conditions for detailed study. In our last study, we 
succeeded in realizing the artificial initiation of the bag-breakup phenomenon in the modified TSWiWaT. Arterially produced bag-breakup phenomenon turned out to be close to that observed under natural conditions of the wind-wave interaction (see the comparison on Figure 2).
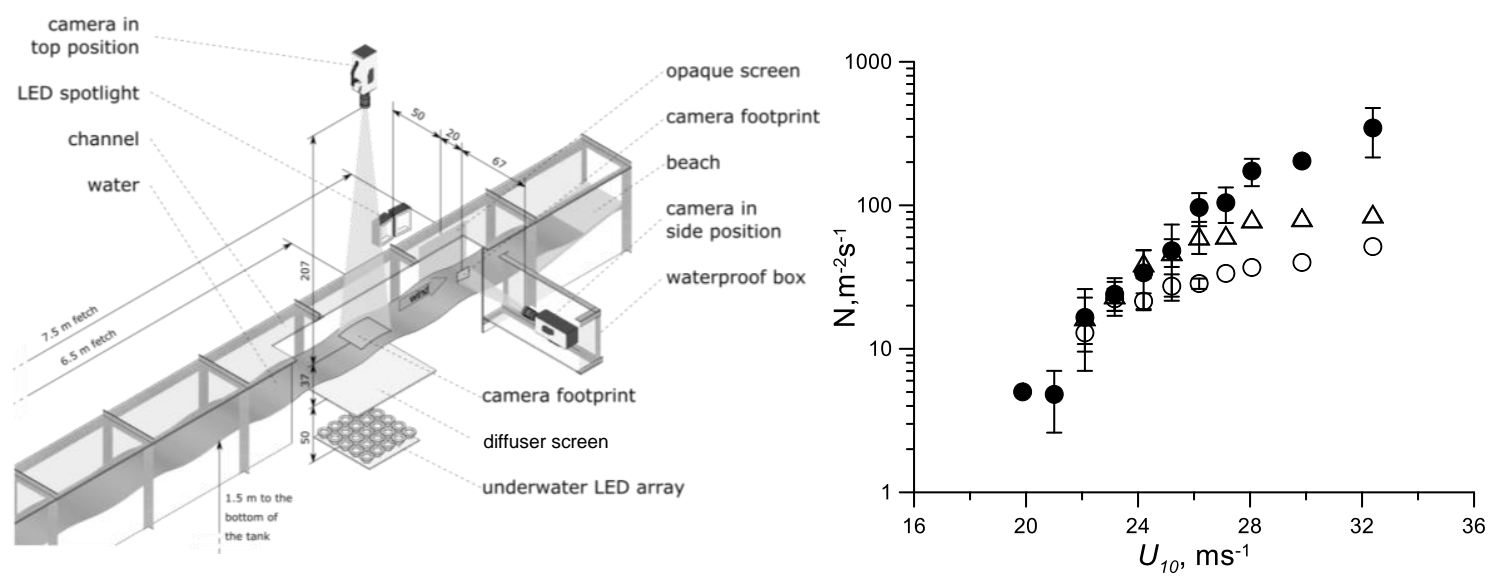

Figure 1: a) Schematic diagram of experimental setup on TSWiWaT, b) Dependence of the specific numbers (per unit time from unit surface) of the spray-generating phenomena on the wind speed. Open circles indicate bursting of floating bubbles, triangles the liquid filaments, and fill circles the bag breakup events is adapted from [11].
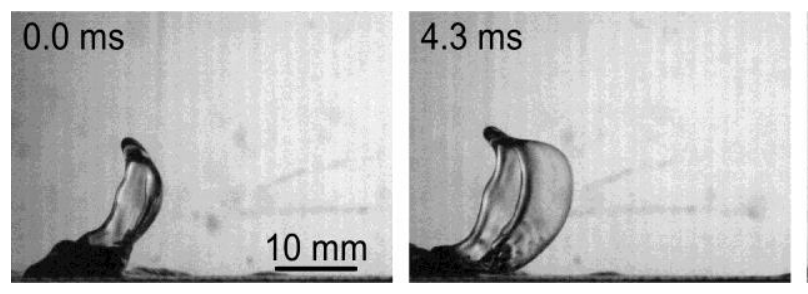

(a)

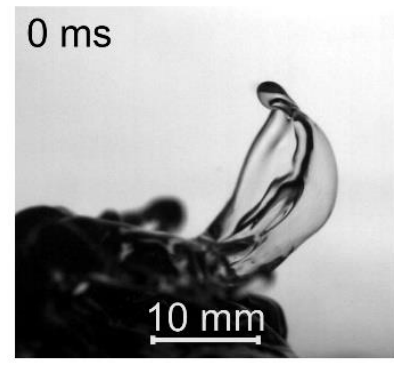

$4 \mathrm{~ms}$

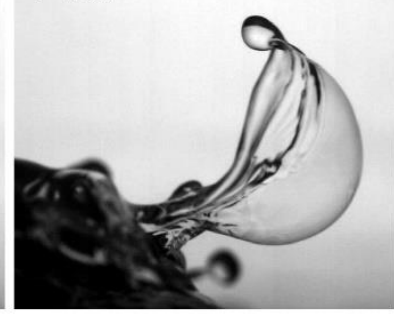

(b)
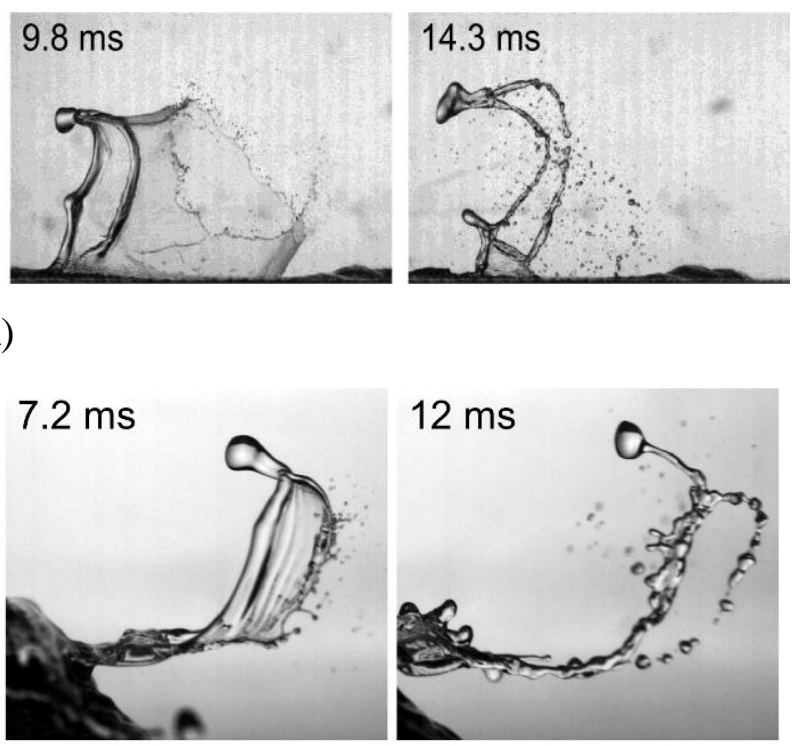

Figure 2: Comparison of the images a) bag-breakup on the real wavy surface b) artificially induced bagbreakup

\section{Methods of laboratory modelling and measurements phenomena of foam coverage over the water surface}

Foam (or whitecapping), like spray, is almost always present on the sea surface, with the exception of only very weak waves and wind. In its characteristics, it is very different from both water and air, which determines its special role in the processes of exchange between the atmosphere and the sea surface. The main mechanism of the foam coverage formation is concerned with intensive breaking of the wave crests which leads to underwater air involvement and formation of the bubbles and subsequent whipping into the foam. Large-scale near-surface currents, for example, Langmuir circulation also can 
lead to the foam coverage formation. By combining various factors, the relative area of the foam coverage increases sharply in hurricane winds $\left(U_{10}\right.$ more than $\left.30 \mathrm{~m} / \mathrm{s}\right)$ and exceeds $70 \%$ (the results presented in [11]). It should be noted that field data are characterized by a large scatter. Uncertainties complicate use of field data for development of theoretical models. Therefore, in the present work, a system for the artificial generation of foam was first developed, as well as a technique for measuring the characteristics of the foam (relative coverage area and bubble sizing) specifically for research in laboratory modeling.

Experiments to study the characteristics of the foam in the framework of laboratory modeling were carried out also at the TSWiWaT (see [12]). In conditions of clean water, as well as small accelerations, it is difficult to ensure regular generation of foam. Investigations of the foam parameters in the framework of laboratory experiments were also carried out on TSWiWaT (see [12]). In clear water conditions, as well as short fetches, it is difficult to ensure regular foaming. Therefore, to create a stable foam coverage of the required density on the water surface, a special device (foam generator) was developed. It includes two diffusers (tubes $1 \mathrm{~cm}$ in diameter and $350 \mathrm{~mm}$ long and with side holes 2 $\mathrm{mm}$ in diameter with a pitch of $7 \mathrm{~mm}$ ) connected by side surfaces to each other, covered with foam rubber. The device was positioned horizontally, perpendicular to the flume, at a depth of less than 5 $\mathrm{mm}$, on the distance from the airflow inlet is $1.2 \mathrm{~m}$. The foaming agent solution is supplied through one of the diffusers under a pressure of $1.5 \mathrm{~m}$ high level, compressed air (1.5 Barr) is supplied through the second diffuser to push the foaming agent solution through the foam layer. As a result, finely dispersed foam forms on the surface of the water. The result is finely dispersed foam coverage on the water surface. The level of the foam solution agent and the compressed air pressure were maintained on the fix level for all experiments. It is ensured a constant level of the foam coverage formation rate regardless of the air flow rate and wave parameters

The foam coverage parameters were measured on the distance of $6.5 \mathrm{~m}$ from the inlet for six different wind speeds $U_{10}$ from 12 to $34 \mathrm{~m} / \mathrm{s}$. We used the shadow method of visualizing processes on the rough surface of the water with backlight under the water, which has proven itself in studying the statistics of spray generation phenomena (see previous paragraph).

The number of pixels which of lower light intensity than a certain threshold was used to estimate the proportion of surface area under the foam coverage. The original images were divided into regions, and for each region average intensity was calculated. Such approach of averaging was used eliminate the influence of noise and small optical inhomogeneities.

One background image was calculated as the average for each pixel of the image, using control records taken without wind. After subtracting the background image for each frame, histogram common for all frames was constructed. It is the distribution of the areas of all images in terms of brightness (intensity). The histogram allows us to estimate the proportion of areas with intensity below the threshold, which depends on the level of threshold set.

To determine the threshold, a direct calculation of the foam coverage area using morphological image processing was performed. It was carried out in several stages shown on Figure 3 a-f. The background was subtracted from the original image and cropping was carried out; the image was binarized with a fixed threshold; the morphological opening with the structural element "disk" of $1 \mathrm{px}$ was performed followed by the morphological closure with a "disk" of $21 \mathrm{px}$; closed regions of the image were filled with flood-fill operation. The ratio of the number of pixels found to the total number of pixels was taken as the fraction of the surface area covered with foam.

Based on this analysis, a relative coverage was found for sequences of frames from several records in experiments with artificial foam at $U_{10}=12 \mathrm{~m} / \mathrm{s}$. For each record, a relative brightness threshold is selected for analysis by histograms, which gives the corresponding coverage. Then this threshold value averaged over several over all realizations for $U_{10}=12 \mathrm{~m} / \mathrm{s}$ was used for all cases of wind speeds. Information about the typical size of the foam bubbles was obtained additionally to data on the area of foam coverage. For this purpose, several frames were selected (for each record), on which the foam is clearly visible. Areas were identified on which the bubbles merge (located in several layers) and others unsuitable for automatic determination of the area. For the remaining areas of the images after the preliminary filtering, all circles were found (see Figure $3 \mathrm{~g}$ ) by two-stage circular Hough transform (see [13]) and their distribution in radius was calculated. 


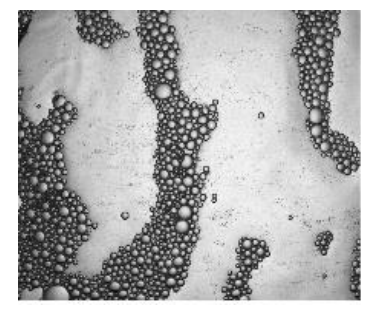

(a)

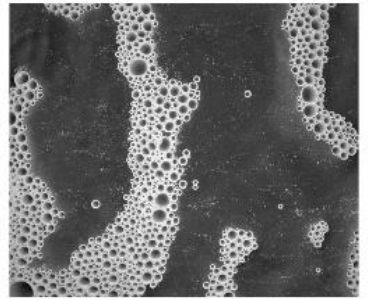

(b)

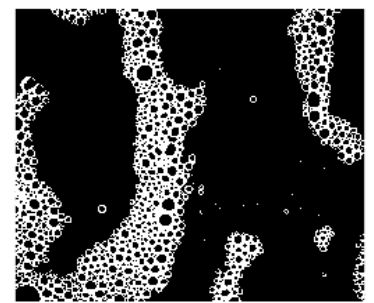

(c)

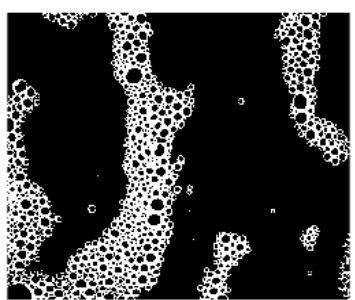

(d)

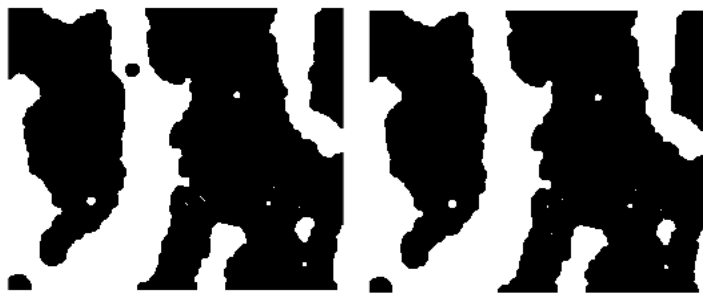

(e) (f)

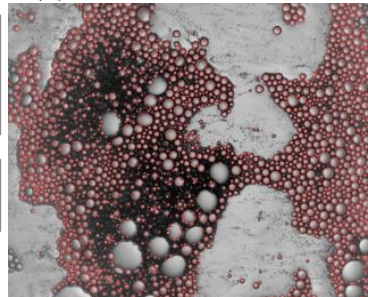

(g)

Figure 3: (a-f) sequences of stages of morphological analysis of the images to determine the relative foam coverage (g) an example of bubble determination.

\section{Acknowledgements}

Investigations were perforned out under support of Russian Science Foundation project 19-17-00209 (development of an optical system) and project 21-19-00755 (development of software for image processing and carrying out experiments), participation of A. Kandaurov was supported by the President's grant for young scientists MK-5503.2021.1.5, participation of A.Suvorov was supported within state assignment of IAP RAS (Theme No. 0030-2019-0018). The experiments were carried out at unique scientific facility "Complex of large-scale geophysical facilities of IAP RAS".

\section{References}

[1] L. Cavaleri, B. Fox-Kemper, M. Hemer, Wind Waves in the Coupled Climate System, Bulletin of the American Meteorological Society 93(11) (2012) 1651-1661. doi.org/10.1175/BAMS-D-1100170.1

[2] F. Veron, Ocean Spray, Annu. Rev. Fluid Mech. 47 (2015) 507-538. doi.org/10.1146/annurevfluid-010814-014651

[3] S. Komori, et al., Laboratory Measurements of Heat Transfer and Drag Coefficients at Extremely High Wind Speeds, J. Phys. Oceanogr. 48 (2018) 959-974. doi.org/10.1175/JPO-D-17-0243.1

[4] R. J. Lai, O. H. Shemdin, Laboratory study of the generation of spray over water, J. Geophys. Res. 79 (1974) 3055-3063. doi.org/10.1029/JC079i021p03055

[5] J. Wu, Spray in the atmospheric surface layer: Laboratory study, J. Geophys. Res. 78 (1973) $511-$ 519. doi.org/10.1029/JC078i003p00511

[6] C. W. Fairall, M. L. Banner, W. L. Peirson, W. Asher, R. P. Morison, Investigation of the physical scaling of sea spray spume droplet production, J. Geophys. Res. 114 (2009) C10001. doi.org/10.1029/2008JC004918

[7] M. Anguelova, R. P. Barber, Wu, Laboratory observation of spume drops produced by wind tearing of wave crests, J. Phys. Oceanogr. 29 (1999) 1156-1165. doi:10.1175/15200485(1999)029<1156:SDPBTW>2.0.CO;2

[8] M. Koga, Direct production of droplets from breaking wind-waves - its observation by a multicolored overlapping exposure photographing technique, Tellus 33 (1981) 552-563. doi.org/10.3402/tellusa.v33i6.10776

[9] Y. I. Troitskaya, O. S. Ermakova, A. A. Kandaurov, D. S. Kozlov, D. A. Sergeev, S. S. Zilitinkevich, Fragmentation of the 'bag-breakup' type as a mechanism of the generation of sea spray at strong and hurricane winds, Dokl. Earth Sci. 477(1) (2017) 1330-1335. doi.org/10.1134/S1028334X17110174 
[10] Y. I. Troitskaya, Y.I. Troitskaya, O.S. Ermakova, A.A. Kandaurov, D.A. Sergeev, D.S. Kozlov, S.S. Zilitinkevich, Non-monotonous dependence of the ocean surface drag coefficient on the hurricane wind speed due to the fragmentation of the ocean-atmosphere interface, Doklady Earth Sciences 477 (2017) 1373-1378. doi.org/10.1134/S1028334X17110265

[11] L. H. Holthuijsen, M. D. Powell, J. D. Pietrzak, Wind and waves in extreme hurricanes, J. Geophys. Res. 117 (2012). C09003, doi:10.1029/2012JC007983.

[12] Yu Troitskaya, D. Sergeev, A. Kandaurov, M. Vdovin, S.S. Zilitinkevich, J. Physical. Ocean., The Effect of Foam on Waves and the Aerodynamic Roughness of the Water Surface at High Winds 49(4) (2019), 959-981. doi.org/10.1175/JPO-D-18-0168.1

[13] H.K. Yuen, J. Princen, J. Illingworth, and J. Kittler. Comparative study of Hough transform methods for circle finding, Image and Vision Computing. 8(1) (1990), 71-77. doi:10.1016/02628856(90)90059-E 